\title{
Design of College Students English Contest System Based on NET Platform
}

\author{
Jin Chun $^{1}$, Xing Ruonan ${ }^{2}$ a \\ ${ }^{1}$ Foreign Languages School, Jiangxi Science \& Technology Normal University, Nanchang, \\ 330000, China \\ ${ }^{2}$ Communication School, Jiangxi Normal University, Nanchang, 330000, China \\ ayjgnine@163.com
}

Keywords: English Contest; Management System; ASP.Net; Workflow

\begin{abstract}
With the development of information technology, the Information Management System of the English Contest for College Students is presented to strengthen the Management Information. By analyzing the management content and workflow of the English Contest for College Students, integrated network technology, workflow and database, the Information Management System of the English Contest for College Students is designed and implemented on the ASP platform. Through the system, the relevant information of the English contest can be revealed and the paperless workflow management of the contest will be realized in the process of application and endorsement as well as online registration.
\end{abstract}

\section{Introduction}

As for promoting the overall improvement of the college students' English level and arousing the college students' interest in learning English, the English contest is an important carrier, which plays a crucial role in cultivating college students' comprehensive quality and innovative ability. The domestic educational administrative departments at all levels and universities increasingly enlarge investment in the English contest and actively encourage college students to participate in the contest, aimed to improve the construction of the study style in the universities and stimulate students' motivation for learning. Due to the cumbersome management process of the contest and too many approval procedures, there exist some problems like work inefficiency and data errors in the application of traditional manual management approach. Based on the ASP.Net platform, the management system of the English Contest for College Students allows the management of the English contest to be networked and automated. Not only can this reduce the workload and errors of the managerial personnel, but also save a great amount of material and financial resources, which increases the efficiency of the management to some extent.

\section{System Development Technology}

ASP Technology. ASP means dynamic server pages. It is an application developed by Microsoft to replace the CGI scripts as well as a simple and convenient programming tool, which can interact with databases and other programs. As open non-compiled application software, ASP enables users to utilize the powerful functions of HTML and Active $\mathrm{X}$ to create a robust, powerful and platform-independent Web application system. The format of ASP page files is .asp, which is commonly used in various dynamic websites. [1]ASP is a kind of server-side scripting environment that can be used to create or run dynamic Web pages or Web applications. ASP can contain HTML tags, ordinary texts, script commands and COM components. ASP can be used to add interactive contents to the pages and also HTML pages can be created as Web application with user interfaces.[2]

NET Technology. NET is a new generation of computer software development platform based on open Internet standards and protocols to achieve a high degree of interactivity in heterogeneous language and platform, designed to simplify the development environment of the applications under 
the highly-distributed environment of the third generation of Internet. [3]NET combines the most outstanding computing and communication schemes, such as XML, SOAP and other Internet standards. Such integration of standards and schemes will contribute to the development from the simple message based on HTML protocol to the programmable information based on XML standards. NET Framework is the foundation of NET platform and meanwhile Microsoft Corporation links its strategic objectives for future development closely with NET framework. Therefore, NET platform will be one of the widely-used software development platforms. This study concentrates on the development of the management system of the English Contest for College Students, which is based on NET platform, allowing the management of the English contest to be networked and automated. Not only can this reduce the workload and errors of the managerial personnel, but also save a great amount of material and financial resources, which increases the efficiency of the management.

\section{System Design}

System Demand Analysis. The management system of the English contest is designed to solve the problems existing in the current manual management of the English contest in the school. For example, the management process is cumbersome with low work efficiency; participants and data information are of such large quantity that errors are prone to occur; the declaration of the funds of participation and the filling of the feedback after winning the prizes are not standardized, affecting the level of management; before, during and after the contest, the information is not timely published, not conducive to the internal and external publicity. In order to solve the problems mentioned above, quantities of investigation as the basis and the needs of all the departments combined, the main demands of the system are determined as follows:

Design of System Functions. According to the preliminary demand analysis, function modules of the system are designed. The system is a management platform for the English Contest for College Students, combining the announcement of the contest information, the declaration and approval of the contest, the display of the awards, and the confirmation of the awards after the contest and other functions as a whole. The function structure chart is shown as Figure 1:

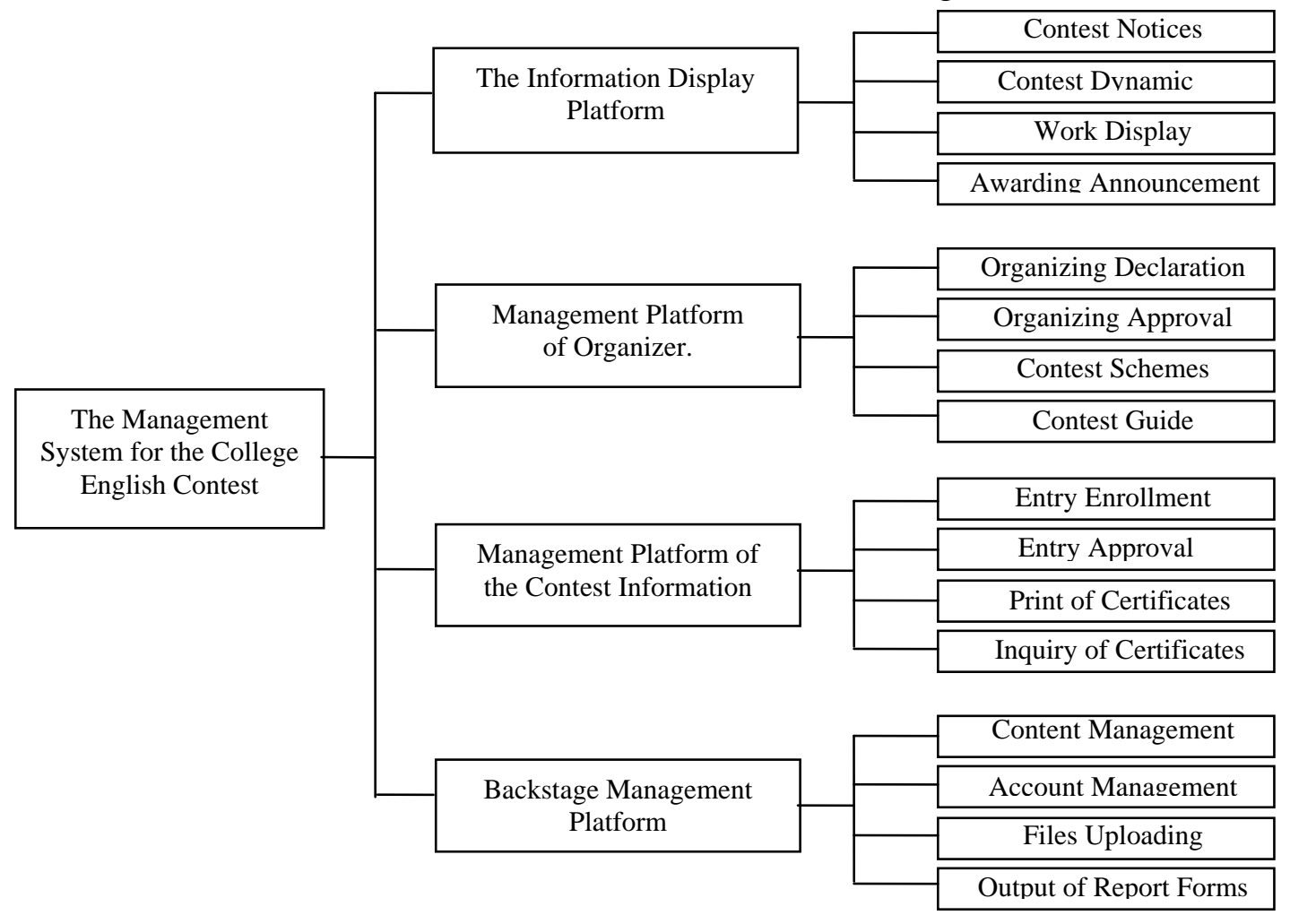

Fig. 1. function structure chart of the Management System for College English Contest 


\section{System Implementation}

System Implementation Technology. Based on Visual Studio 2008 integrated development platform, the development of the system takes the ASP.NET and C\#.NET as the programming language, SQLAERVER 2008 as the backstage database and NET Workflow as the workflow engine, achieving the integration of network technology, workflow technology and database technology. [4]The system is based on B/S network structure mode with the server-side operation system being Windows 2003 Server. As long as the client end is equipped with the browser with the version IE 6.0 or above, the system can be operated, which is at low cost, easy to maintain and convenient for upgrading. The software programming method adopts the three-tier design, the presentation layer, business layer and data layer respectively, making the structure of the system clearer and the divisions explicit, which is beneficial for the later maintenance and upgrade. The presentation encapsulates the client end, providing the access to the application programs, namely the ASP.NET page in the system; the business layer encapsulates the business function of the system, using the C\# language to realize the business logic; the data layer encapsulates the function of data access using the ADO.NET data access method, providing services for the business logic layer. The three-tier structure figure for the system implementation technology shows as Figure 2:

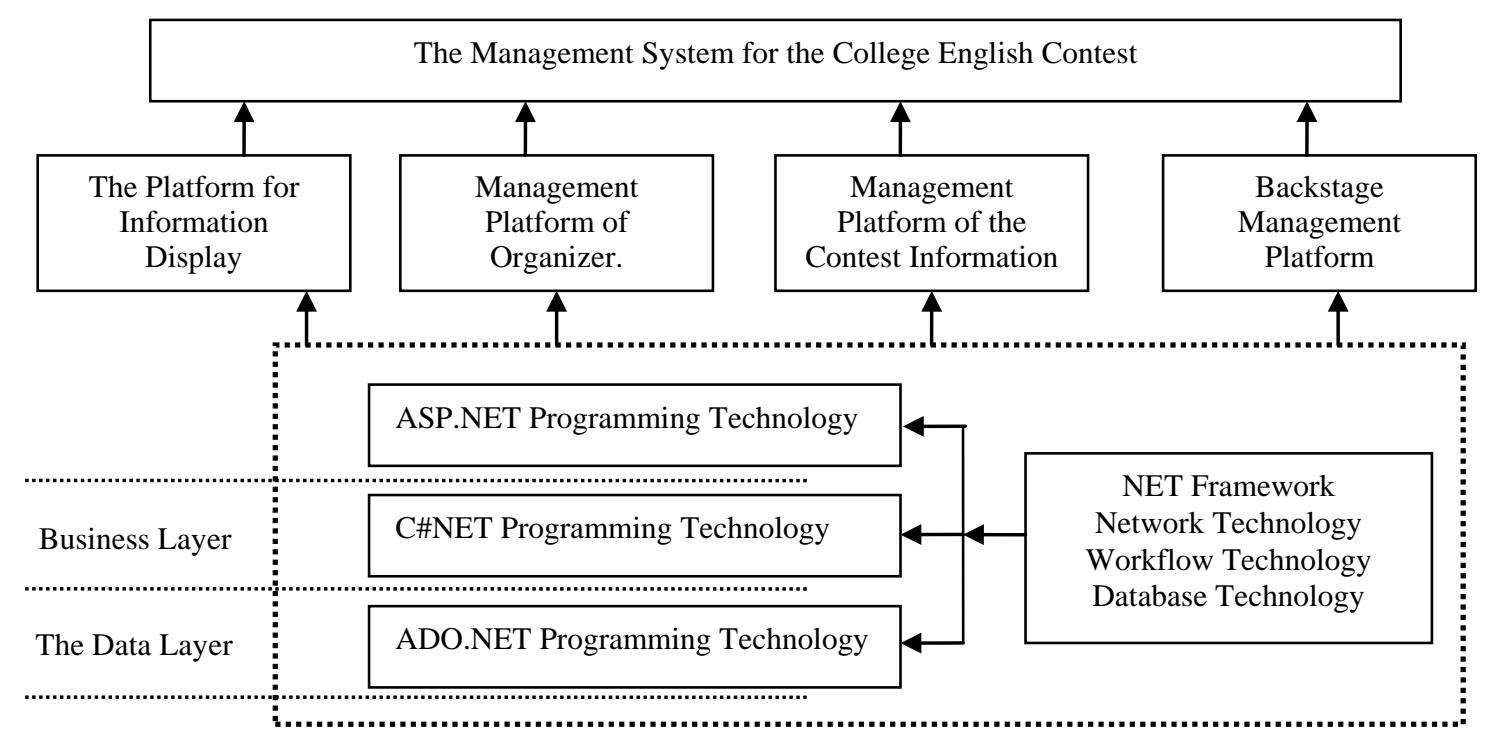

Fig. 2. the three-tier structure figure for the system implementation technology

The Function Modules of the System Implementation Technology. From a structural and functional point of view, the B/S mode applied by the group contest management system, in the logical architecture, can be divided into the three-tier architecture: the presentation layer, business logic layer and database layer. [5]Under normal conditions, the client end does not directly interact with database, but establish a connection with business logic layer and then interact with database through it.

(1) The user layer that lies at the client end. Used to realize the user interface shown in client browser (i.e. Internet Explore and other Web browsers), this layer shows the data information transmitted by the business logic layer in a proper form and then complete the conversation between the client and the application. Meanwhile, this layer is responsible for obtaining and verifying the data input by the users and transferring the data to the business logic layer.

(2) The business logic layer provides the user interface with function calls. The work like business rules, data access and verification of legitimacy is put into the layer for processing. At the same time, this layer is responsible for receiving the requirements from the browser and accessing database by calling the function provided by the data access layer, and finally it transmits the processing results back to the browser. [6] This layer is supposed to structure the crucial objects in the project according to the design of the whole system so as to achieve the major logic control functions of the project. 
(3) The database layer: The layer is on the bottom layer, based on the ADO.NET platform to realize the operation and maintenance of the Microsoft Access 2003 database. It mainly deals with the requirements for data from the business logic layer and implements all the operations on tables in the database, such as adding, modifying and deleting the records. It is through the ADO.NET data adapter and the stored procedures that the operation of database is completed.

The Security Strategy of the System. To ensure the security of the system, the following measures are adopted. According to the practical needs, the users of the system are divided into three types, the backstage management, the organizer and the participants, each type as a specific role endowed with corresponding permissions. Only when the permission of the access or operation of a certain module is gained can the module be accessed or operated. Properties like the users' names and permissions can be modified to maintain, which not only improves the security of the system, but avoids attributing the permission to each user, increase the efficiency and flexibility. [7]

\section{Conclusion}

The Management System of the English Contest for College Students developed now adopts the .NET technology framework, SQL Server 2012 database management technology, C\# .NET development technology and other advanced development technology, completing the design and implementation of the database with the merits of high reliability, high security, scalability, expansibility and reusability. The operation of the system shows that the system enables each link of the management of the English contest to be networked and standardized, reducing the workload of managing the contest to a large extent, saving the manpower and material resources and thus improving the work efficiency of the English contest, which is useful for reference.

\section{References}

[1] Yang Yanlan, Jin Xiaoxue, Ye Hua. Research on Framework of ASP.NET AJAX and Its Application in Web Development . Computer Applications and Software, 2011, 06: pp:195-198.

[2] Huang Shenggen, Chen Shuyu. Design and Implementation of the Leader Education System Based on ASP.NET MVC Framework. Computer Technology and Its Development, 2010, 02,pp:190-193.

[3] Lin Xu. Design and Implementation of Online Examination System Based on ASP.NET[D]. Electronic Science and Technology University,2013.

[4] Cheng Wenwei. ASP.NET Database Development. Journal of Guangzhou University (Natural Science Edition), 2005,02:137-140.

[5] Cao Jianying. The Safety of ASP.NET and Its Implementation Strategy. Journal of Wenzhou Normal College (Natural Science Edition), 2005,02:99-102.

[6] Zhao Chengsong. Design and Implementation of an Educational Administration System Based on .NET Platform. Electronic Science and Technology University,2013.

[7] Yang Jiao. Design and Implementation of Teacher Information Management System Based on ASP.NET. Nanjing University of Posts and Telecommunications,2013. 